\title{
Comparison of Spinal Canal Expansion Following Cervical Laminoplasty Based on the Preoperative Lamina Angle: A Simulation Study
}

\author{
Jong-myung Jung, ${ }_{1}^{1}$ Anthony L. Jahng, ${ }^{2}$ Seung-Jae Hyun, ${ }^{3} \mathrm{Ki}-J e o n g ~ K i m,{ }^{3}$ Tae-Ahn Jahng ${ }^{3}$ \\ Department of Neurosurgery, Spine Center, Gachon University Gil Medical Center, Gachon University College of Medicine, Incheon, Korea \\ College of Arts and Science, ${ }^{2}$ Case Western Reserve University, Cleveland, OH, USA \\ Department of Neurosurgery, ${ }^{3}$ Spine Center, Seoul National University Bundang Hospital, Seoul National University College of Medicine, \\ Seongnam, Korea
}

Objective : Expansion in the spinal canal area (SCA) after laminoplasty is one of the critical factors to relieve the preoperative symptoms. No previous study has compared the increases in SCA achieved by open-door laminoplasty (ODL) and double door laminoplasty (DDL) according to the preoperative lamina angle $(\mathrm{LA})$. This study was designed to clarify the relationship between the laminoplasty opening angle (OA)/laminoplasty opening size (OS) and increases in the SCA following ODL and DDL according to the preoperative LA using a simulation model.

Methods : The simulation model was constructed and validated by comparing the clinical data of 64 patients who had undergone C3-C6 laminoplasty (43 patients with ODL and 21 patients with DDL). SCA expansion was predicted with a verified simulation model at various preoperative LAs (from $28^{\circ}$ to $32^{\circ}$ ) with different OAs $\left(40^{\circ}\right.$ to $\left.44^{\circ}\right)$ and OSs ( $10 \mathrm{~mm}$ to $14 \mathrm{~mm}$ ) recruited from patient data.

Results : The constructed simulation model was validated by comparing clinical data and revealed a very high degree of correlation $(r=0.935, p<0.001)$. In this validated model, at the same OA, the increase in SCA was higher following ODL than following DDL in the usual LA $(p<0.05)$. At the same OS, the increase in SCA was slightly larger following DDL than following ODL, but the difference was not significant $(p>0.05)$. The difference was significant when the preoperative $L A$ was narrower or much wider.

Conclusion : Based on clinical data, a simulation model was constructed and verified that could predict increases in the SCA following ODL and DDL. When applying this model, prediction in SCA increase using the OS parameter was more practical and compatible with clinical data. Both laminoplasties achieved enough SCA, and there was no significant difference between them in the usual range.

Key Words : Cervical vertebrae $\cdot$ Laminoplasty $\cdot$ Spinal canal $\cdot$ Mathematics.

\section{INTRODUCTION}

Surgical treatment of cervical spondylotic myelopathy
(CSM) and ossified posterior longitudinal ligament (OPLL) is performed by either direct removal of the cord-compressing lesion through the anterior approach or indirect decompres-

- Received : June 18, $2020 \bullet$ Revised : July 15, 2020 •Accepted : July 20, 2020

- Address for reprints : Jong-myung Jung

Department of Neurosurgery, Spine Center, Gachon University Gil Medical Center, Gachon University College of Medicine, 21 Namdong-daero 774beon-gil, Namdong-gu, Incheon 21565, Korea

Tel : +82-32-460-3304, Fax : +82-32-460-3899, E-mail : jmjung2625@naver.com, ORCID : https://orcid.org/0000-0002-3718-7740

This is an Open Access article distributed under the terms of the Creative Commons Attribution Non-Commercial License (http://creativecommons.org/licenses/by-nc/4.0) which permits unrestricted non-commercial use, distribution, and reproduction in any medium, provided the original work is properly cited. 
sion through the posterior approach. Cervical laminoplasty is considered a standard technique for posterior decompression, and several types of laminoplasty have been described in recent decades. Current laminoplasty techniques tend to fall into one of two categories : open-door laminoplasty (ODL) or double-door laminoplasty (DDL). Each procedure preserves the lamina but expands the size of the spinal canal by placing the partially freed lamina in a more posterior position to produce a wider spinal canal as this allows a greater chance for the spinal cord to recover its functions. When performing both techniques, the laminoplasty opening size (OS) has the greatest impact on the amount of spinal canal area (SCA) after surgery. Several studies have reported that there is a relationship between OS and $\mathrm{SCA}^{6,20)}$. However, a comparison of the increase in the SCA following ODL and DDL according to the preoperative lamina angle (LA) has rarely been performed. And the range of preoperative LA was limited.

The purpose of this study was to clarify the relationship between the laminoplasty opening angle (OA)/laminoplasty OS and increases in the SCA following ODL and DDL according to the preoperative LA using a simulation model.

\section{MATERIALS AND METHODS}

The present study was approved by the Institutional Review Board of Seoul National University Bundang Hospital (B-
$1705 / 395-107)$

\section{Patient data}

Between January 2012 and December 2016, we treated 157 patients with CSM or OPLL by cervical laminoplasty. Four neurosurgeons performed laminoplasties; three performed ODL, and one performed DDL. Seventy-five patients with C3-C6 laminoplasty were selected. Of these patients, 11 with cervical deformities, trauma, infection, and previous cervical spine surgery were excluded. Ultimately, 64 patients were included in this study, including 43 in the ODL group and 21 in the DDL group. Parameters related to pre- and postoperative computed tomography (CT) findings, including preoperative LA, OA, OS, and increases in SCA, were measured for comparison in the simulation model.

\section{Simulation model construction and validation}

CT scanning was performed in all patients with a slice thickness of $3 \mathrm{~mm}$. Axial CT cuts obtained at each pedicle level from C3 to C6 were used for measurements. The distances between both lamina-facet junctions (from points A to B and from points E to F), the LA $(\alpha)$, the OA $(\beta)$, the OS and the SCA were measured with an accuracy within $0.01^{\circ}$ and $0.01 \mathrm{~mm}$ in the ODL and DDL groups (Fig. 1). All parameters were measured using picture archiving and communication system software (M-view, version 5483; INFINITT Healthcare, Seoul, Korea). Two clinicians independently evaluated all
(A)

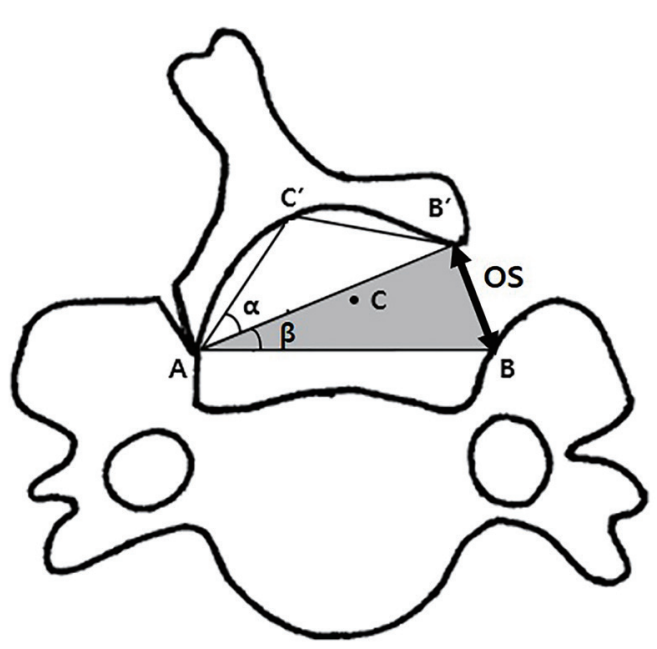

(B)

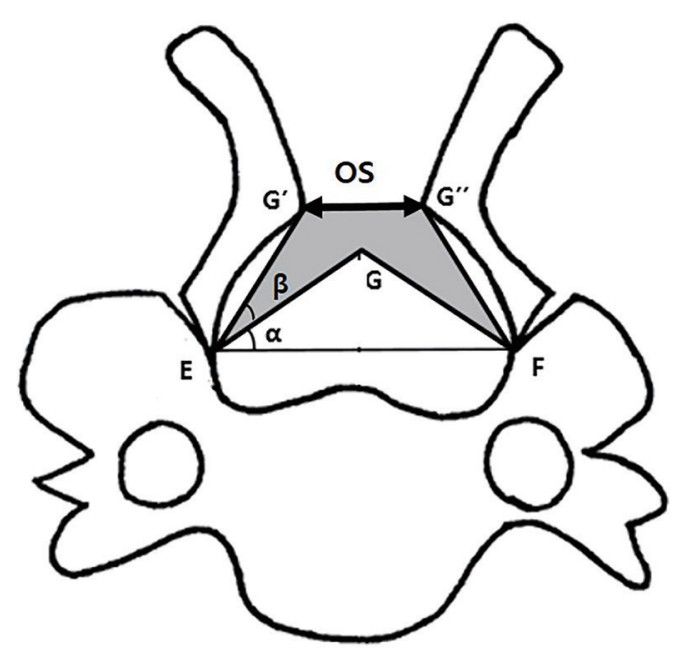

Fig. 1. Radiologic parameters used in the present study. The shaded area shows the increase in the spinal canal area achieved after laminoplasty. A : Open-door laminoplasty. B : Double-door laminoplasty. $\alpha$ indicates the preoperative lamina angle and $\beta$ indicates the laminoplasty opening angle. OS : opening size. 
the images twice, and the mean value was used for subsequent analysis.

GeoGebra software package version 5.0 (International GeoGebra Institute, Linz, Austria) for Windows was used to simulate ODL and DDL (Fig. 2). GeoGebra is a mathematics software program for education that can be used for geometry, algebra, spreadsheets, graphing, statistics and calculus ${ }^{5}$. The program has been used for research in various areas, such as orthopedic trauma, chemistry, veterinary medicine, and environmental management ${ }^{1,3,12,16,18)}$. In this simulated study, we have drawn an isosceles triangle into the cross-sectional image of the spinal canal. Bilateral gutters were created at the transitional portion of the lamina and the lateral mass, and the positions of the gutters were not changed in either the open or double-door procedure. In ODL, points A and B represent the most medial points of the bilateral laminar gutters (Fig. 2). Point $\mathrm{C}$ represents the inner edge of the lamina. Point $\mathrm{B}$ and $\mathrm{C}$ shift back and form points B' and C'. $\alpha$ indicates the preoperative LA, and $\beta$ indicates the laminoplasty OA. In DDL, points $\mathrm{E}$ and $\mathrm{F}$ represent the most medial points of the bilateral laminar gutters. Point $\mathrm{G}$ represents the inner edge of the lamina and shift back and forms point $\mathrm{G}^{\prime}$ and $\mathrm{G}^{\prime \prime}$. For equivalent $\mathrm{OA}$ simulation, $B^{\prime}$ was defined $\operatorname{Rot}\left(B, \beta^{\circ}, A\right)$. It meant rotating point $B$ by $\beta$ degree relative to point $A$ (Fig. 2). Like this, all points have been defined and formulated. The lamina was then rotated around the most medial point of the gutter until a target OA or OS was achieved. Canal expansion with a different colored area could be created after laminoplasty. In this way, the enlarged spinal canal could be calculated.

A validation study was performed to assess the accuracy of the simulation model in reproducing ODL and DDL. Pre- and postoperative CT scans obtained 64 patients who had already undergone either ODL or DDL were analyzed.

\section{Prediction of the increase in the SCA}

Based on the pre- and postoperative parameters of CT findings, laminoplasty with the $\mathrm{OA}$ of $40^{\circ}, 42^{\circ}, 44^{\circ}$ and that with the OS of 10,12 , and $14 \mathrm{~mm}$ were simulated according to preoperative LA.

\section{Statistical analysis}

Statistical analyses were performed using Student's t-test for continuous variables and the chi-square test or Fisher's exact test for categorical variables. A two-sided $p$-value of $<0.05$ was considered significant. The differences between clinical data and simulation results were evaluated with a paired t-test. The correlation between clinical data and simulation results was assessed by calculating Pearson's correlation coefficient. SPSS statistical software package version 21.0 (IBM Corporation, Armonk, NY, USA) for Windows was used for statistical analyses.

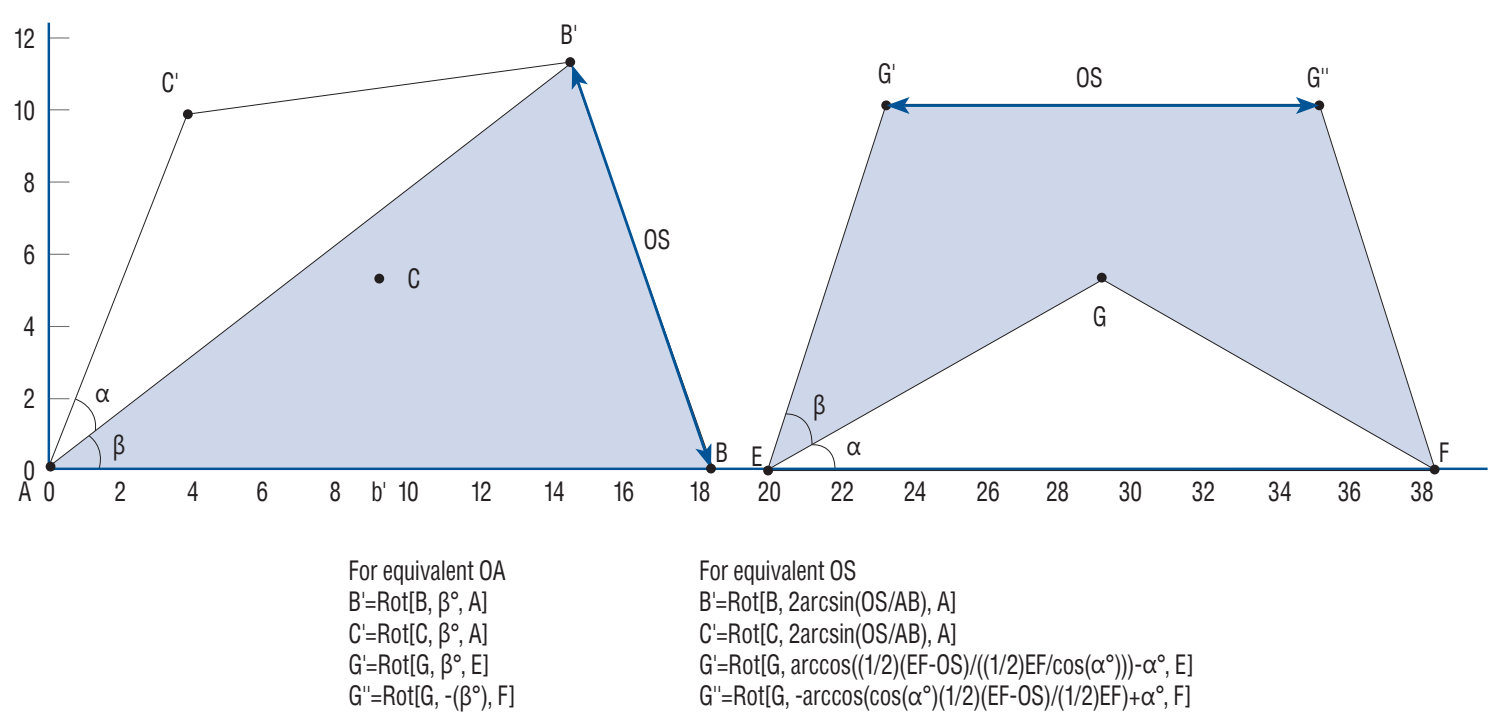

Fig. 2. Images of the simulation model developed using the GeoGebra program (International GeoGebra Institute, Linz, Austria). The shaded area shows the increased area of the spinal canal achieved after laminoplasty. $a$ indicates the preoperative lamina angle and $\beta$ indicates the laminoplasty opening angle. OA : opening angle, OS : opening size. 


\section{RESULTS}

We retrospectively analyzed the clinical and radiologic characteristics of 64 patients (Table 1). The two groups did not differ significantly in age, sex, and diagnosis $(p>0.05)$. Radiologically, there was no significant difference in the distance between both lamina-facet junctions and the preoperative LA $(p>0.05)$ between the ODL and DDL groups. The usual LA at the $\mathrm{C} 3-6$ level of the 64 patients ranged from $28.44^{\circ}$ to $32.39^{\circ}$, while the OA ranged from $40.35^{\circ}$ to $44.43^{\circ}$. The commonly used OS was $12 \mathrm{~mm}$, and $10 \mathrm{~mm}$ and $14 \mathrm{~mm}$ were followed in both groups. These values were applied to the simulation model to predict SCA after both types of laminoplasty.

The constructed simulation model was verified using clinical data. A comparison of the clinical data with the simulation results showed no significant difference $(p>0.05)$ and a very high degree of correlation $(\mathrm{r}=0.935, p<0.001)$ (Table 2). These findings support the validity of the simulation model. Therefore, the accuracy of the simulation model can be considered adequate for the current study.

Although both techniques showed that the SCA increased at an $\mathrm{OA}$ of $40^{\circ}, 42^{\circ}$, or $44^{\circ}$, each of which was a typical OA in

Table 1. Demographic and radiographic parameters of the 64 patients

\begin{tabular}{|c|c|c|c|}
\hline & ODL $(n=43)$ & $\operatorname{DDL}(n=21)$ & $p$-value \\
\hline Age (years) & $63.3 \pm 12.0$ & $59.9 \pm 7.5$ & 0.17 \\
\hline Sex, male : female & $35: 8$ & $16: 5$ & 0.63 \\
\hline Diagnosis, CSM : OPLL & $10: 33$ & $6: 15$ & 0.65 \\
\hline C3 distance between both LFJ (mm) & $19.24 \pm 1.44$ & $18.72 \pm 1.12$ & 0.28 \\
\hline Preoperative lamina angle $\left(^{\circ}\right)$ & $32.39 \pm 1.44$ & $31.60 \pm 2.21$ & 0.76 \\
\hline C4 distance between both LFJ (mm) & $19.13 \pm 1.08$ & $18.73 \pm 1.59$ & 0.36 \\
\hline Preoperative lamina angle $\left(^{\circ}\right)$ & $28.89 \pm 1.75$ & $28.44 \pm 2.37$ & 0.89 \\
\hline C5 distance between both LFJ (mm) & $18.67 \pm 0.95$ & $18.55 \pm 1.14$ & 0.45 \\
\hline Preoperative lamina angle $\left(^{\circ}\right)$ & $30.75 \pm 1.72$ & $29.58 \pm 2.71$ & 0.69 \\
\hline C6 distance between both LFJ (mm) & $18.85 \pm 0.53$ & $18.23 \pm 1.70$ & 0.20 \\
\hline Preoperative lamina angle $\left(^{\circ}\right)$ & $29.71 \pm 2.05$ & $29.29 \pm 2.66$ & 0.12 \\
\hline
\end{tabular}

Values are presented as mean \pm standard deviation unless otherwise indicated. ODL : open-door laminoplasty, DDL : double-door laminoplasty, CSM : cervical spondylotic myelopathy, OPLL : ossification of the posterior longitudinal ligament, LFJ : lamina-facet junctions

Table 2. Comparison of the clinical data obtained by computed tomography and those obtained using the simulation results predicted by the GeoGebra program (paired t-test and Pearson correlation analysis)

\begin{tabular}{|c|c|c|c|c|}
\hline Level & Clinical data $\left(\mathrm{mm}^{2}\right)$ & Simulation results $\left(\mathrm{mm}^{2}\right)$ & t value ( $p$-value) & r value ( $p$-value) \\
\hline \multicolumn{5}{|c|}{ Increase in the SCA following ODL } \\
\hline C3 & $101.99 \pm 8.93$ & $97.21 \pm 6.72$ & $1.49(0.13)$ & $0.954\left(<0.001^{*}\right)$ \\
\hline C4 & $105.07 \pm 9.23$ & $101.89 \pm 7.63$ & $1.74(0.61)$ & $0.910\left(<0.001^{*}\right)$ \\
\hline $\mathrm{C} 5$ & $105.60 \pm 9.43$ & $102.96 \pm 7.23$ & $1.93(0.81)$ & $0.940\left(<0.001^{*}\right)$ \\
\hline $\mathrm{C} 6$ & $107.18 \pm 7.37$ & $103.19 \pm 6.03$ & $1.13(0.20)$ & $0.910\left(<0.001^{*}\right)$ \\
\hline \multicolumn{5}{|c|}{ Increase in the SCA following DDL } \\
\hline C3 & $105.79 \pm 9.41$ & $100.02 \pm 7.97$ & $1.44(0.61)$ & $0.919\left(<0.001^{*}\right)$ \\
\hline $\mathrm{C} 4$ & $106.91 \pm 9.51$ & $102.77 \pm 7.02$ & $1.93(0.81)$ & $0.939\left(<0.001^{*}\right)$ \\
\hline C5 & $110.56 \pm 7.37$ & $106.37 \pm 6.14$ & $1.44(0.61)$ & $0.959\left(<0.001^{*}\right)$ \\
\hline $\mathrm{C} 6$ & $110.78 \pm 6.35$ & $107.62 \pm 5.56$ & $1.72(0.12)$ & $0.949\left(<0.001^{*}\right)$ \\
\hline
\end{tabular}

Values are presented as mean \pm standard deviation unless otherwise indicated. ${ }^{*}$ Indicates statistical significance. SCA : spinal canal area, ODL : opendoor laminoplasty, DDL : double-door laminoplasty 
the patients recruited in this study, the SCA was significantly larger following ODL than it was following DDL $(p<0.05)$ (Table 3). The increase in SCA after ODL was not affected by preoperative LA when OA was fixed to a certain angle. The increase in SCA was smaller for DDL than for ODL in the usual range of $\mathrm{OA}$, but it gradually increased as the preoperative LA or OA increased. When the OA increased to over the typical range, the increase in SCA was larger in DDL than in ODL (Fig. 3).

A commonly used OS was between 10 and $14 \mathrm{~mm}$ based on the clinical data presented here. When the OS was between 10 and $14 \mathrm{~mm}$, the increase in the SCA following ODL was constant at each preoperative LA (Table 4) and gradually increased as OS increased. Within the same OS range, the increase in SCA following DDL was slightly larger than that observed following ODL at each preoperative angle, but the difference was not significant. These increases in DDL tended to gradually decrease as preoperative LA increased in the same OS range. In simulations performed outside the usual range of preoperative LA, the difference of increase in SCA observed between $28^{\circ}$ and $42^{\circ}$ was not significant following either type of laminoplasty, although the increase was larger for DDL than for ODL (Fig. 4). Outside of these ranges, DDL resulted in significantly larger increases than were achieved by ODL when the LA was either narrower or wider.

\section{DISCUSSION}

The overlying goals of management for CSM and OPLL are cord decompression, stabilization, preservation and improvement of cord vascularity, and restoration of normal sagittal alignment. Many factors must be considered when determining the procedure needed to achieve the aforementioned goals of management adequately. Several factors, such as cervical curvature, OPLL, anterior bony spur, K-line, must be taken into account when planning ${ }^{2)}$.

A variety of potential prognostic factors may affect clinical

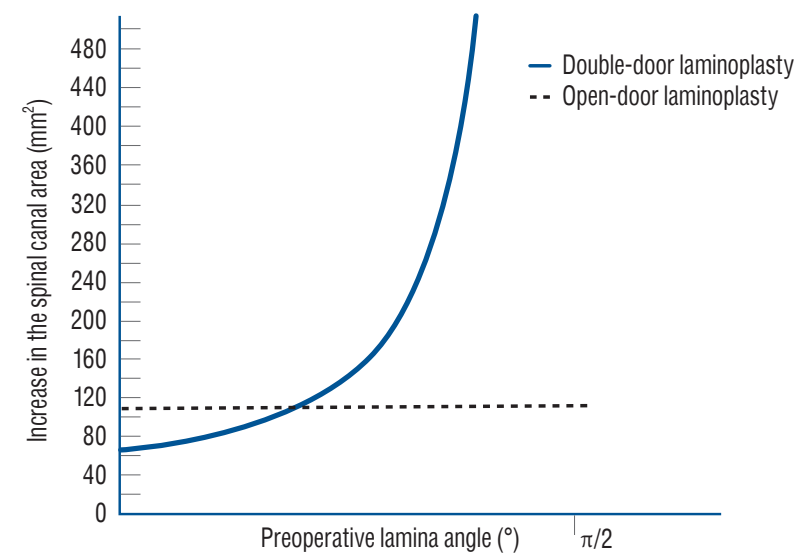

Fig. 3. Increases in the spinal canal area after open-door and double-door laminoplasty according to the preoperative lamina angle under same opening angle.

Table 3. Increase in the spinal canal area at laminoplasty OAs of $40-44^{\circ}$ for preoperative LAs of $28-32^{\circ}$

\begin{tabular}{|c|c|c|c|c|c|c|}
\hline \multirow{2}{*}{$\operatorname{LA}\left({ }^{\circ}\right)$} & \multicolumn{2}{|c|}{$O A$ of $40^{\circ}$} & \multicolumn{2}{|c|}{$\mathrm{OA}$ of $42^{\circ}$} & \multicolumn{2}{|c|}{$\mathrm{OA}$ of $44^{\circ}$} \\
\hline & $\mathrm{ODL}\left(\mathrm{mm}^{2}\right)$ & $\mathrm{DDL}\left(\mathrm{mm}^{2}\right)$ & $\mathrm{ODL}\left(\mathrm{mm}^{2}\right)$ & $\mathrm{DDL}\left(\mathrm{mm}^{2}\right)$ & ODL $\left(\mathrm{mm}^{2}\right)$ & $\mathrm{DDL}\left(\mathrm{mm}^{2}\right)$ \\
\hline $28^{\circ}$ & $108.34 \pm 7.31$ & $94.64 \pm 14.12$ & $112.78 \pm 7.31$ & $99.83 \pm 14.12$ & $117.08 \pm 14.13$ & $104.97 \pm 15.59$ \\
\hline$p$-value & \multicolumn{2}{|c|}{$<0.05^{*}$} & \multicolumn{2}{|c|}{$<0.05^{*}$} & \multicolumn{2}{|c|}{$<0.05^{*}$} \\
\hline $29^{\circ}$ & $108.34 \pm 7.31$ & $96.34 \pm 7.32$ & $112.78 \pm 7.31$ & $101.58 \pm 7.32$ & $117.08 \pm 14.13$ & $106.77 \pm 8.11$ \\
\hline$p$-value & \multicolumn{2}{|c|}{$<0.05^{*}$} & \multicolumn{2}{|c|}{$<0.05^{*}$} & \multicolumn{2}{|c|}{$<0.05^{*}$} \\
\hline $30^{\circ}$ & $108.34 \pm 7.31$ & $98.11 \pm 10.28$ & $112.78 \pm 7.31$ & $103.42 \pm 10.28$ & $117.08 \pm 14.13$ & $108.65 \pm 12.71$ \\
\hline p-value & \multicolumn{2}{|c|}{$<0.05^{*}$} & \multicolumn{2}{|c|}{$<0.05^{*}$} & \multicolumn{2}{|c|}{$<0.05^{*}$} \\
\hline $31^{\circ}$ & $108.34 \pm 7.31$ & $99.97 \pm 16.75$ & $112.78 \pm 7.31$ & $105.33 \pm 16.75$ & $117.08 \pm 14.13$ & $110.62 \pm 18.15$ \\
\hline p-value & \multicolumn{2}{|c|}{$<0.05^{*}$} & \multicolumn{2}{|c|}{$<0.05^{*}$} & \multicolumn{2}{|c|}{$<0.05^{*}$} \\
\hline $32^{\circ}$ & $108.34 \pm 7.31$ & $101.92 \pm 11.11$ & $112.78 \pm 7.31$ & $107.34 \pm 11.11$ & $117.08 \pm 14.13$ & $112.68 \pm 12.14$ \\
\hline$p$-value & \multicolumn{2}{|c|}{$<0.05^{*}$} & \multicolumn{2}{|c|}{$<0.05^{*}$} & \multicolumn{2}{|c|}{$<0.05^{*}$} \\
\hline
\end{tabular}

Values are presented as mean \pm standard deviation. ${ }^{*}$ Indicates statistical significance. OA : opening angle, LA : lamina angle, ODL : open-door laminoplasty, DDL : double-door laminoplasty 
Table 4. Increase in the spinal canal area at laminoplasty OSs of $10-14 \mathrm{~mm}$ for preoperative LAs of $28-32^{\circ}$

\begin{tabular}{|c|c|c|c|c|c|c|}
\hline \multirow{2}{*}{$\operatorname{LA}\left({ }^{\circ}\right)$} & \multicolumn{2}{|c|}{ OS of $10 \mathrm{~mm}$} & \multicolumn{2}{|c|}{ OS of $12 \mathrm{~mm}$} & \multicolumn{2}{|c|}{ OS of $14 \mathrm{~mm}$} \\
\hline & $\mathrm{ODL}\left(\mathrm{mm}^{2}\right)$ & $\mathrm{DDL}\left(\mathrm{mm}^{2}\right)$ & $\mathrm{ODL}\left(\mathrm{mm}^{2}\right)$ & $\mathrm{DDL}\left(\mathrm{mm}^{2}\right)$ & $\mathrm{ODL}\left(\mathrm{mm}^{2}\right)$ & $\mathrm{DDL}\left(\mathrm{mm}^{2}\right)$ \\
\hline $28^{\circ}$ & $88.59 \pm 6.12$ & $90.44 \pm 6.73$ & $104.43 \pm 7.41$ & $105.76 \pm 8.25$ & $119.19 \pm 8.78$ & $120.04 \pm 9.85$ \\
\hline$p$-value & \multicolumn{2}{|c|}{ NS } & \multicolumn{2}{|c|}{ NS } & \multicolumn{2}{|c|}{ NS } \\
\hline $29^{\circ}$ & $88.59 \pm 6.12$ & $90.06 \pm 7.83$ & $104.43 \pm 7.41$ & $105.43 \pm 9.72$ & $119.19 \pm 8.78$ & $119.77 \pm 11.63$ \\
\hline$p$-value & \multicolumn{2}{|c|}{ NS } & \multicolumn{2}{|c|}{ NS } & \multicolumn{2}{|c|}{ NS } \\
\hline $30^{\circ}$ & $88.59 \pm 6.12$ & $89.73 \pm 7.72$ & $104.43 \pm 7.41$ & $105.15 \pm 9.54$ & $119.19 \pm 8.78$ & $119.55 \pm 11.38$ \\
\hline$p$-value & \multicolumn{2}{|c|}{ NS } & \multicolumn{2}{|c|}{ NS } & \multicolumn{2}{|c|}{ NS } \\
\hline $31^{\circ}$ & $88.59 \pm 6.12$ & $89.43 \pm 6.07$ & $104.43 \pm 7.41$ & $104.91 \pm 7.38$ & $119.19 \pm 8.78$ & $119.38 \pm 8.83$ \\
\hline$p$-value & \multicolumn{2}{|c|}{ NS } & \multicolumn{2}{|c|}{ NS } & \multicolumn{2}{|c|}{ NS } \\
\hline $32^{\circ}$ & $88.59 \pm 6.12$ & $89.18 \pm 5.62$ & $104.43 \pm 7.41$ & $104.71 \pm 6.83$ & $119.19 \pm 8.78$ & $119.26 \pm 8.27$ \\
\hline$p$-value & \multicolumn{2}{|c|}{ NS } & \multicolumn{2}{|c|}{ NS } & \multicolumn{2}{|c|}{ NS } \\
\hline
\end{tabular}

Values are presented as mean \pm standard deviation. OS : opening size, LA : lamina angle, ODL : open-door laminoplasty, DDL : double-door laminoplasty, NS : not significant

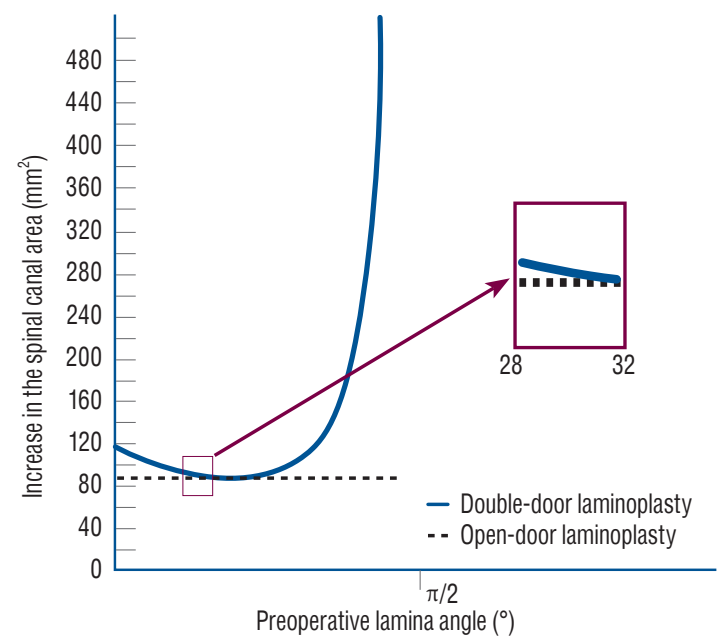

Fig. 4. Increases in the spinal canal area after open-door and doubledoor laminoplasty according to the preoperative lamina angle under same opening size. The inserted figure shows a magnified view for preoperative lamina angles ranging from $28^{\circ}$ to $32^{\circ}$.

outcomes after cervical laminoplasty ${ }^{21}$. Spinal canal expansion is one of the critical factors to affect clinical results after cervical laminoplasty. Especially concerning spinal canal expansion, there is a positive correlation between the likelihood of functional spinal cord recovery and the degree of spinal cord decompression. In cases of inadequate increases in the sagittal canal diameter or SCA, spinal cord compression will not be relieved, resulting in unsatisfactory results after cervical laminoplasty ${ }^{9,11,19)}$. The minimal extent to which the spinal canal must be widened to obtain good results remains unclear, although the relationship between the degree of spinal canal expansion and clinical outcomes after laminoplasty has been investigated. Other studies have reported that the optimal amount the spinal canal must be increased in size after cervical laminoplasty is over $4-5 \mathrm{~mm}$ in the sagittal diameter ${ }^{10,13)}$. Regarding the increase in SCA, widening the area by $95 \mathrm{~mm}^{2}$ achieved good functional recovery in one study, while another study reported that patients with an SCA of $\geq 160 \mathrm{~mm}^{2}$ after cervical laminoplasty made better clinical outcomes ${ }^{8,13)}$. Excessive openings of the lamina may cause the cord to migrate and extend posteriorly to an excessive degree, which can lead to the occurrence of postoperative C5 nerve root palsy ${ }^{11)}$. The excessive opening also creates a wider epidural space and induces the formation of more epidural scar tissues than would normally be expected ${ }^{19)}$. Although other prognostic factors are predetermined before laminoplasty, the degree of spinal canal expansion is adjustable. Hence, surgeons can decide to expand the canal by different amounts based on how long the spacers are, making it indeed an on-demand procedure.

Many researchers have studied canal expansion after ODL and DDL. A cadaveric study showed that both laminoplasty techniques are effective at increasing the surface area of the spinal canal without any significant difference ${ }^{4)}$. While some clinical studies have reported finding no significant difference in radiologic results between ODL and DDL, other clinical studies have found that the magnitude of spinal canal expansion was larger in the ODL group than in the DDL group ${ }^{14,15,17,22}$. Park et 
al. ${ }^{17)}$ reported that the SCA expanded from $204.3 \pm 4.15$ to 331.7 $\pm 5.58 \mathrm{~mm}^{2}$ in the ODL group and from $201.2 \pm 8.26$ to $280.8 \pm$ $20.92 \mathrm{~mm}^{2}$ in the DDL group $(p<0.001)$. In another study by Nakashima et al. ${ }^{15)}$, the rate of spinal canal enlargement was also significantly higher in the ODL group than in the DDL group (ODL group, 256.6\% $\pm 77.6 \%$; DDL group, $213.5 \% \pm$ $69.8 \% ; p=0.007)$. However, in our clinical data, DDL resulted in more SCA expansion at every level, indicating that SCA expansion does not merely depend on the type of laminoplasty and that it instead depends on the length of the spacer, the OA, and the location of the hinge and lateral gutter (Table 2).

Few studies have been performed to predict SCA expansion using simulation modeling or computerized programs ${ }^{6,7,19)}$. Wang et al. ${ }^{19)}$ used a computer-simulated comparison to predict spinal canal expansion following cervical laminoplasty. They concluded that the differences between postoperative canal increases achieved by ODL versus DDL were statistically significant when the door was opened by more than $12 \mathrm{~mm}$ $(p<0.05)$. Unfortunately, they did not consider different preoperative LAs, and their results, therefore, did not reflect the effects of changes in the preoperative LA. Gu et al. ${ }^{6}$ reported a simulation study of ODL performed to clarify the relationship between the laminoplasty OS and the laminoplasty OA and predict the amount of canal enlargement during ODL. They utilized an image analysis program to predict values, but their calculation formula was too complicated to use in clinical practice to predict SCA expansion, and the authors concluded that a laminoplasty OS of $10-12 \mathrm{~mm}$ at C3-C7 might be optimal during ODL. Furthermore, they did not perform an analysis of SCA changes according to preoperative LA, which can affect SCA expansion in laminoplasty. The same group reported using a similar program in DDL and concluded that it was feasible for predicting SCA expansion ${ }^{7}$. They suggested that SCA expansion was proportional to the preoperative LA and OS but did not provide data regarding the differences between ODL and DDL according to preoperative LA.

In this study, we constructed a simulation model to compare ODL and DDL, validated it using clinical data, and showed that it provided a reliable correlation. SCA expansion was compared according to OA and OS parameters for each laminoplasty at each preoperative LA. When OA parameters were applied, ODL resulted in more SCA expansion than DDL did for the usual preoperative LA ranges (from $28^{\circ}$ to $32^{\circ}$ ). In terms of increasing SCA, ODL had an advantage over DDL in patients with usual preoperative LA or typical OA. A wider OA may be required to obtain similar results in DDL. In fact, in the real patient data included in this study for model verification, the increases in SCA were larger in DDL than ODL, and these results were not compatible with the simulation data obtained using the OA parameter (Tables 2 and 3). This discrepancy may be related to the larger spacer size used in DDL and the narrower OA in ODL in real patients. Surgeons do not measure or determine the necessary OA in the operative field, but instead determine the OS, which is usually similar to the size of the space between lamina. Thus, OS is a more practical parameter for predicting increases in SCA after laminoplasty. Applying OS parameters produced a better correlation in the clinical data, indicating that DDL increases SCA more. When the OS was between 10 and $14 \mathrm{~mm}$, the SCA consistently increased with LA following ODL and gradually increased with OS. Within the same OS range, DDL produced a slightly larger increase in SCA than ODL did at each preoperative angle, but the difference was not significant. For the same OS range, these increases in DDL tended to decrease as preoperative LA increased gradually. We also simulated increases in SCA for preoperative LA values outside the usual ranges. Outside of usual ranges (less than $28^{\circ}$ or more than $42^{\circ}$ ), DDL appeared to increase SCA more. Although DDL could be a better surgical option for expanding the SCA if the LA is much broader or narrower than usual, our simulation study revealed there was no significant difference in SCA changes following either laminoplasty technique within the usual preoperative LA range.

\section{Limitations of the present study}

There are limitations to this study. First, the current simulation model is a simplified standard model and did not analyze the effects of anatomical variation, such as asymmetry, deformation of the spinal canal, and different hinge/lateral gutter locations. Furthermore, this model is not sufficient to reflect every surgical detail or several modifications that can be applied when using either type of laminoplasty. In particular, the increase in the SCA observed after laminoplasty may differ from the simulation results depending on the position of the plate or spacer. Nevertheless, results obtained using the model can be verified with clinical data and can predict the increase in SCA that will be achieved after either laminoplasty technique, especially when using OS parameters. 
Second, this simulation model cannot correlate with clinical outcome data. The clinical outcomes following laminoplasty are related to several factors, such as preoperative neurological grade, the degree of compression, the surgical approach, and postoperative events. An increase in the SCA is one of these factors. Thus, achieving an increase in SCA does not necessarily predict better clinical outcomes. An inadequate or excessive opening may lead to undesirable results after laminoplasty as an inadequate canal opening will not be large enough to decompress the cord and will, therefore, fail to relieve the symptoms. An excessive lamina opening can also cause problems, such as root retraction due to the tethering effect. Thus, it might be necessary to investigate the relationship between the degree of SCA expansion/OS and clinical outcomes associated with preoperative LA.

\section{CONCLUSION}

The current simulation model was verified based on clinical data and can predict the increase in the SCA following ODL and DDL. When applying this model, prediction in SCA increase using the OS parameter was more practical and compatible with clinical data. Although DDL may be a better option in patients with a narrower or wider preoperative LA, both laminoplasties achieved a large enough SCA in the simulation model, and the difference between the two was not significant in the usual range.

\section{CONFLICTS OF INTEREST}

No potential conflict of interest relevant to this article was reported.

\section{INFORMED CONSENT}

This type of study does not require informed consent.

\section{AUTHOR CONTRIBUTIONS}

\author{
Conceptualization : ALJ, TAJ
}

\author{
Data curation : JMJ \\ Formal analysis : JMJ \\ Methodology : JMJ, TAJ \\ Project administration : TAJ \\ Visualization : JMJ \\ Writing - original draft : JMJ \\ Writing - review \& editing : JMJ, SJH, KJK, TAJ
}

\section{ORCID}

Jong-myung Jung https://orcid.org/0000-0002-3718-7740

Anthony L. Jahng https://orcid.org/0000-0003-1674-8593

Seung-Jae Hyun https://orcid.org/0000-0003-2937-5300

Ki-Jeong Kim https://orcid.org/0000-0001-8547-8545

Tae-Ahn Jahng https://orcid.org/0000-0001-8342-3874

\section{References}

1. Bachhal V, Saini G, Jindal N, Sament R, Dadra A : GeoGebra: a reliable and free software for measuring acetabular cup anteversion on digitalized plain radiographs. J Clin Orthop Trauma 11(Suppl 2) : S201S205, 2020

2. Cho SK, Kim JS, Overley SC, Merrill RK : Cervical laminoplasty: indications, surgical considerations, and clinical outcomes. J Am Acad Orthop Surg 26 : e142-e152, 2018

3. De Felice E, Pacioni C, Tardella FM, Dall'Aglio C, Palladino A, Scocco P : A novel method for increasing the numerousness of biometrical parameters useful for wildlife management: roe deer mandible as bone model. Animals (Basel) $10: 465,2020$

4. Deol GS : Comparison of spinal canal expansion between two techniques of cervical laminoplasty. 31st Annual Meeting of Cervical Spine Research Society; 2003 Dec 11-13; Scottsdale, AZ

5. GeoGebra : GeoGebra software. Available at: https://www.geogebra.org/

6. Gu Z, Zhang A, Shen Y, Li F, Sun X, Ding W : Relationship between the laminoplasty opening size and the laminoplasty opening angle, increased sagittal canal diameter and the prediction of spinal canal expansion following open-door cervical laminoplasty. Eur Spine J 24 : 1613-1620, 2015

7. Gu ZF, Zhang AL, Shen Y, Ding WY, Li F, Sun XZ : The relationship between laminoplasty opening angle and increased sagittal canal diameter and the prediction of spinal canal expansion following double-door cervical laminoplasty. Eur Spine J 24 : 1597-1604, 2015

8. Hamburger C, Büttner A, Uhl E : The cross-sectional area of the cervical spinal canal in patients with cervical spondylotic myelopathy. Correlation of preoperative and postoperative area with clinical symptoms. Spine 
(Phila Pa 1976) 22 : 1990-1994; discussion 1995, 1997

9. Hatta $Y$, Shiraishi T, Hase H, Yato $Y$, Ueda S, Mikami Y, et al. : Is posterior spinal cord shifting by extensive posterior decompression clinically significant for multisegmental cervical spondylotic myelopathy? Spine (Phila Pa 1976) 30 : 2414-2419, 2005

10. Hirabayashi K, Watanabe K, Wakano K, Suzuki N, Satomi K, Ishii Y : Expansive open-door laminoplasty for cervical spinal stenotic myelopathy. Spine (Phila Pa 1976) 8 : 693-699, 1983

11. Imagama S, Matsuyama Y, Yukawa Y, Kawakami N, Kamiya M, Kanemura T, et al. : C5 palsy after cervical laminoplasty: a multicentre study. J Bone Joint Surg Br 92 : 393-400, 2010

12. Jovanov $D$, Vujić $B$, Vujić $G$ : Optimization of the monitoring of landfill gas and leachate in closed methanogenic landfills. J Environ Manage $216: 32-40,2018$

13. Kohno K, Kumon Y, Oka Y, Matsui S, Ohue S, Sakaki S : Evaluation of prognostic factors following expansive laminoplasty for cervical spinal stenotic myelopathy. Surg Neurol 48 : 237-245, 1997

14. Naito M, Ogata K, Kurose S, Oyama M : Canal-expansive laminoplasty in 83 patients with cervical myelopathy. A comparative study of three different procedures. Int Orthop 18 : 347-351, 1994

15. Nakashima H, Kato F, Yukawa Y, Imagama S, Ito K, Machino M, et al. : Comparative effectiveness of open-door laminoplasty versus Frenchdoor laminoplasty in cervical compressive myelopathy. Spine (Phila Pa 1976) 39 : 642-647, 2014
16. Pantazi A, Doukakis S : An educational scenario for the learning of the conic section: studying the ellipse with the use of digital tools and elements of differentiated instruction and cognitive neurosciences. Adv Exp Med Biol 1194 : 31-40, 2020

17. Park JH, Roh SW, Rhim SC, Jeon SR : Long-term outcomes of 2 cervical laminoplasty methods: midline splitting versus unilateral single door. J Spinal Disord Tech 25 : E224-E229, 2012

18. Velázquez-Galván $Y$, Encinas $A$ : Analytical magnetostatic model for $2 \mathrm{D}$ arrays of interacting magnetic nanowires and nanotubes. Phys Chem Chem Phys 22 : 13320-13328, 2020

19. Wang $X Y$, Dai $L Y, X u H Z$, Chi YL : Prediction of spinal canal expansion following cervical laminoplasty: a computer-simulated comparison between single and double-door techniques. Spine (Phila Pa 1976) 31 : 2863-2870, 2006

20. Yang XJ, Tian RJ, Su X, Hu SB, Lei W, Zhang Y : Relationship of actual laminoplasty opening size and increment of the cross-sectional area based on single-door cervical laminoplasy. Medicine (Baltimore) 97 : e0216, 2018

21. Yoon ST, Raich A, Hashimoto RE, Riew KD, Shaffrey Cl, Rhee JM, et al. : Predictive factors affecting outcome after cervical laminoplasty. Spine (Phila Pa 1976) 38(22 Suppl 1) : S232-S252, 2013

22. Yue WM, Tan CT, Tan SB, Tan SK, Tay BK : Results of cervical laminoplasty and a comparison between single and double trap-door techniques. J Spinal Disord 13 : 329-335, 2000 